\title{
Long Term Stability Of Metal Oxide-Based Gas Sensors For E-nose Environmental Applications: an overview
}

\author{
Anne-Claude ROMAIN, Jacques NICOLAS \\ University of Liège - Department "Environmental Sciences and Management", \\ Avenue de Longwy, 185, 6700 ARLON - Belgium \\ acromain@ulg.ac.be
}

\begin{abstract}
The e-nose technology has enormous potentialities for in site monitoring of malodors. However a number of limitations are associated with the properties of chemical sensors, the performances of the signal processing and the realistic operation conditions of environmental field. From the experience of the research group in the field, the metal oxide based gas sensors (Figaro type) are until now the best chemical sensors for long term application, more than one year of continuous working in the field. To be usable for malodors measurement in the field, the e-nose has to deal with the lack of long term stability of these sensors. The drift and the sensors replacement have to be considered. In order to appraise the time evolution of the sensors and the effect on the results of an electronic nose, experimentation has been performed during three years on two identical sensor arrays. The two arrays contain the same six Figaro sensors and are in the same sensor chamber of the e-nose system. Both arrays have worked continuously during three years without break.
\end{abstract}

Keywords: e-nose, tin oxide sensors, drift, environmental application.

PACS: 07.88.+y

\section{INTRODUCTION}

The Artificial olfaction system is a very promising tool to monitor the malodor in the field. Usual measurement techniques of odor use human olfaction or conventional analytical techniques. The first category represents the real odor perception but is not applicable to measure continuously bad odors in the field. The second class of techniques gives the mixture composition but not the global information representative of the odor perception. The e-nose has the potentialities to combine "the odor perception" and the "monitoring in the field". The instrument, based on non-specific gas chemical sensors array combined with a chemometric processing tool provides a suitable technique for in site monitoring of malodors. The research group in Arlon has more than ten years experience in the measure of environmental malodors in the field. Published studies report attractive results $[1,2]$. This technique has probably the best potentialities to answer to the expectations of the various actors of the environmental problems in relation with the odors annoyance [3]. However a number of limitations are associated with the properties of chemical sensors the performances of the signal processing and the realistic operation conditions of environmental field. From the experience of the research group in the field, the metal oxide based gas sensors are the best chemical sensors for long term application, more than one year of continuous working. However, as a result of harsh environmental conditions, of hardware limitations and of olfactory pollution specificities, odour real-time monitoring with an electronic nose is always a real challenge. The instrument has to cope with several specific drawbacks. In particular, it has to automatically compensate the time drift [4] and the influence of ambient parameters such as temperature or humidity [5]. This paper is focused on the time drift and the long term stability of the metal oxide gas sensors (Figaro sensors). Sensor drift is a first serious impairment of chemical sensors. The sensors alter over time and so have poor repeatability since they produce different responses for the same odour. That is particularly troublesome for electronic noses [6]. The sensor signals can drift during the learning phase [7]. Another frequent problem encountered in the field and particularly in highly polluted atmosphere is a sensor failure or an irreversible sensor poisoning. Clearly, life expectancy of sensors is reduced for real-life operation with respect to clean lab operation. Sensor replacement is generally required to address such issue, but, after 
replacement, odours should still be recognised without having to recalibrate the whole system. But commercial sensors are rarely reproducible.

\section{EXPERIMENTAL AND METHODS}

In order to appraise the time evolution of the sensors and the effect on the results of an electronic nose, experimentation was performed during three years on two identical sensor arrays. The two arrays contain the same six Figaro sensors (see Table 1) and are in the same sensor chamber of the e-nose system. Both arrays worked continuously during three years without break.

TABLE 1. the six selected Figaro sensors.

\begin{tabular}{cc}
\hline Sensor & $\begin{array}{c}\text { Application (from the } \\
\text { manufacturer) }\end{array}$ \\
\hline TGS 822 & Organic solvents (ethanol, benzene, \\
acetone, ..) \\
TGS 880 & $\begin{array}{c}\text { Volatiles vapour from food } \\
\text { (alcohols)x }\end{array}$ \\
TGS 842 & Natural gas, methanex \\
TGS 2610 & Propane, butane \\
TGS 2620 & Hydrogen, alcohols, organic solvents \\
TGS 2180 & water \\
\hline
\end{tabular}

To try to compensate the sensor drift, three types of solutions were tested for our applications. The usual way of minimising drift effect is to consider as useful response the difference between the base line, obtained by presenting the sensor array to pure reference air, and the signal obtained after stabilisation in the polluted atmosphere. However, such solution requires operating by cycling between reference air and tainted air, which is not convenient for on-site applications. That requires carrying in the field heavy gas cylinders. Alternatively, generating the reference air by a simple filtering of ambient atmosphere gives rise to only partial drift compensation and to a lack of purity of the reference gas, which increase the data dispersion. Posterior global drift counteraction algorithms could be applied either for each individual sensor or by correcting the whole pattern, using multivariate methods. First the main direction of the drift is determined in the first component space of the multivariate method, such as Principal Component Analysis (PCA), or by selecting time as dependant variable of a Partial Least Square regression (PLS). The drift component can then be removed from the sample gas data, correcting thus the final score plot of the multivariate method [8].n order to appraise the time evolution of the sensors and the effect on the results of an electronic nose, experimentation was performed during three years on two identical sensor arrays. The two arrays contain the same six Figaro sensors (see Table 1) and are in the same sensor chamber of the e-nose system. Both arrays worked continuously during three years without break.

\section{RESULTS}

\subsection{Drift}

Figure 1 shows drift compensation of a commercial tin oxide sensor (TGS2620, FigaroTM) by multiplicative factor estimated from calibration measurements.

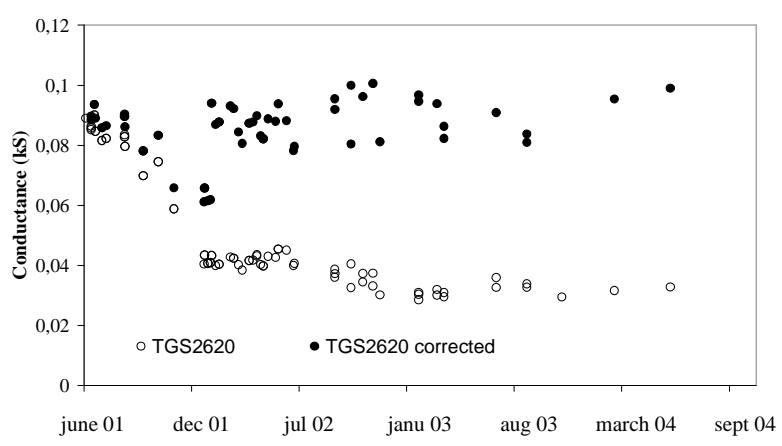

FIGURE 1. Drift correction of the sensor TGS 2620 by multiplicative factor estimated by calibration gas measurements.

The multiplicative factor seems to be able to compensate the drift of this sensor.

In order to test the performance of the method with this calibration gas on malodour measurements, linear discriminant analysis (LDA or fisher discriminant analysis) is applied for two different environmental odours: compost odour and printing house odour. Measurements are obtained with an array of 5 tin oxide sensors.

Linear discriminant analysis (LDA) was used with 5 features (5 sensor conductance values) and 63 observations collected within a 22- month's period. Two classes were considered (compost class and printing house class) The Fisher-Snedecor F-ratio of intergroup/intragroup variances was chosen as classification performance criterion. Higher the $F$ value is, better is the separation of the classes.

TABLE 2. Evaluation of the classification without correction or with correction models (by the $\mathrm{F}$ criterion, F-ratio of intergroup/intragroup variances)..

\begin{tabular}{lc}
\hline \multicolumn{1}{c}{ Method } & F \\
\hline No correction & 33 \\
$\begin{array}{l}\text { Correction by sensor (individual } \\
\text { multiplicative factor) }\end{array}$ & 56 \\
Correction of the sensor array "PLS" & 26 \\
Correction of the sensor array "PCA" & 18
\end{tabular}




\subsection{Sensor replacement}

Figure 2 shows a PCA score-plot in the plane of the two first components. It concerns 260 observations, 3 classes (ethanol, background air and compost odour), 5 features and a 2-year period. After the replacement of the sensors in the array with the same trade mark references, the previous calibrated model is no longer applicable for the same odorous emissions: all the observation points are shifted to another part of the diagram.

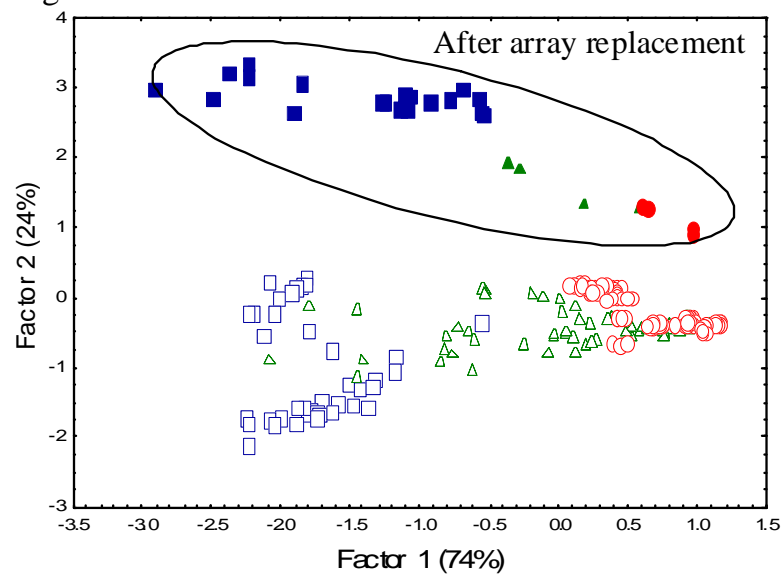

FIGURE 2. Illustration, by a PCA score plot of the shift of the measurements after the replacement of the TGS sensors

Again, correction routines including algorithms for handling shift related to sensor replacement can be successfully applied. For the above example, illustrated in figure 2 , the classification performances were severely reduced after array replacement. The percentages of correct classification were $40 \%, 100 \%$ and $33 \%$ respectively for ethanol vapour, background air and compost emission.

After individual sensor correction (with the same procedure individual multiplicative sensor than for drift correction), each classification rate reaches $100 \%$ (table 3).

TABLE 3. Percentage of correct classification after replacement of sensors with and without correction

\begin{tabular}{lcc}
\hline \multicolumn{1}{c}{ Source } & \multicolumn{2}{c}{ \% of correct classification } \\
\cline { 2 - 3 } & $\begin{array}{c}\text { Without } \\
\text { correction }\end{array}$ & $\begin{array}{c}\text { With } \\
\text { correction }\end{array}$ \\
\hline Ethanol & 40 & 100 \\
Air & 100 & 100 \\
Compost & 33 & 100
\end{tabular}

\section{CONCLUSIONS}

Univariate sensor correction gave the best results for complex data like malodours measurements.
With real-life measurements, it is indeed very difficult to identify a single direction in a multivariate space that is only correlated to sensor drift. So, for each sensor, an individual multiplicative factor was calculated by estimating the drift slope for a standard gas.

To meet the requirements of this environmental use of artificial olfaction system, the signal processing method must be simple, but not simplistic, and capable of generalisation. It must be tolerant to hardware weaknesses and adapted for application in the real life.

\section{REFERENCES}

1. Bourgeois, W., Romain, A.-C., Nicolas, J., Stuetz, R. M., "The use of sensor arrays for environmental monitoring: interests and limitations," J. Environ. Monit., Vol. 5, No. 6 (2003), pp. 852-860

2. Romain, A.-C., Nicolas, J., Wiertz, V., Maternova, J., Andre, P., "Use of a simple tin oxide sensor array to identify five malodours collected in the field," Sensors and Actuators B: Chemical, Vol. 62, No. 1 (2000), pp. 73-79.

3. Romain, A.-C., Delva, J., Nicolas, J., "Complementary approaches to measure environmental odours emitted by landfill areas," Sensors and Actuators B: Chemical, Vol. 131, No. 1 (2008), pp. 18-23.

4. Romain, A.-C., Andre, P., Nicolas, J., "Three years experiment with the same tin oxide sensor arrays for the identification of malodorous sources in the environment," Sensors and Actuators B: Chemical, Vol. 84, No. 2-3 (2002), pp. 271-277.

5. Romain, A.-C., Nicolas, J., Andre, P., "In situ measurement of olfactive pollution with inorganic semiconductors : Limitations due to humidity and temperature influence," Seminars in Food Analysis, Vol. 2, (1997), pp. 283-296.

6. Romain, A.-C., Nicolas, J., "Three years experiment with the same tin oxide sensor arrays for the identification of malodorous sources in the environment," Sensors and Actuators B: Chemical, Vol. 84 (2002), pp. 271-277.

7. Holmberg, M., Davide, F. A. M., Di Natale, C., D'amico, A., Winquist, F., Lundstrom, I., "Drift counteraction in odour recognition applications: Lifelong calibration method" Sensors and Actuators B: Chemical, Vol. 42, No. 3 (1997), pp. 185-194.

8. Artursson, T., Eklov, T., Lundström, I., Martersson, P., Sjöström, M., Holmberg, M., "Drift correction for gas sensors using multivariate methods", Journal of chemometrics, Vol. 14, (2000), pp. 711-723. 